\title{
The Cascading Analysts Algorithm
}

\author{
Matthias Ruhl \\ Accompani Inc \\ Los Altos, CA, USA \\ matthias@accompani.com
}

\author{
Mukund Sundararajan* \\ Google Research \\ Mountain View, CA, USA \\ mukunds@google.com
}

\author{
Qiqi Yan \\ Google Research \\ Mountain View, CA, USA \\ contact@qiqiyan.com
}

\section{ABSTRACT}

We study changes in metrics that are defined on a cartesian product of trees. Such metrics occur naturally in many practical applications, where a global metric (such as revenue) can be broken down along several hierarchical dimensions (such as location, gender, etc).

Given a change in such a metric, our goal is to identify a small set of non-overlapping data segments that account for a majority of the change. An organization interested in improving the metric can then focus their attention on these data segments.

Our key contribution is an algorithm that naturally mimics the operation of a hierarchical organization of analysts. The algorithm has been successfully applied within Google's ad platform (AdWords) to help Google's advertisers triage the performance of their advertising campaigns, and within Google Analytics to help website developers understand their traffic.

We empirically analyze the runtime and quality of the algorithm by comparing it against benchmarks for a census dataset. We prove theoretical, worst-case bounds on the performance of the algorithm. For instance, we show that the algorithm is optimal for two dimensions, and has an approximation ratio $\log ^{d-2}(n+1)$ for $d \geq 3$ dimensions, where $n$ is the number of input data segments. For the advertising application, we can show that our algorithm is a 2-approximation.

To characterize the hardness of the problem, we study data patterns called conflicts. These allow us to construct hard instances of the problem, and derive a lower bound of $1.144^{d-2}$ (again $d \geq 3$ ) for our algorithm, and to show that the problem is NP-hard; this justifies are focus on approximation.

\section{CCS CONCEPTS}

- Information systems $\rightarrow$ Data analytics; • Applied computing $\rightarrow$ Decision analysis;

${ }^{*}$ Corresponding Author

Permission to make digital or hard copies of part or all of this work for personal or classroom use is granted without fee provided that copies are not made or distributed for profit or commercial advantage and that copies bear this notice and the full citation on the first page. Copyrights for third-party components of this work must be honored. For all other uses, contact the owner/author(s).

SIGMOD'18, June 10-15, 2018, Houston, TX, USA

(C) 2018 Copyright held by the owner/author(s).

ACM ISBN 978-1-4503-4703-7/18/06.

https://doi.org/10.1145/3183713.3183745

\section{KEYWORDS}

explanation; business metrics; decision support; business intelligence

\section{ACM Reference Format:}

Matthias Ruhl, Mukund Sundararajan, and Qiqi Yan. 2018. The Cascading Analysts Algorithm. In SIGMOD'18: 2018 International Conference on Management of Data, June 10-15, 2018, Houston, TX, USA. ACM, New York, NY, USA, 14 pages. https: //doi.org/10.1145/3183713.3183745

\section{MOTIVATION}

Organizations use metrics to track, analyze, and improve the performance of their businesses. The organization might be a company, a government, an advertiser or a website developer. And the metric might be the revenue of the company, the level of unemployment in a country, or the number of clicks for an online advertising campaign. Indeed, our interest in this problem stems from creating tools that help analysts reason about Google's revenue, to help Google's advertisers reason about the performance of their advertising campaigns, and to help website developers analyze changes in traffic patterns to their website.

Metrics vary because of changes in the business environment. One common task of a data scientist is to determine what drives changes in a metric over time. In particular, they want to identify segments of the business where the change is most pronounced. This helps decision-makers within the organization to counter these changes if they are negative, or amplify the changes if they are positive.

Example 1.1. Consider a government data scientist analyzing an increase in unemployment. She does this by comparing the current month's employment data to the previous month's data to figure out what caused the increase.

The domain of this data - the employment market - can be sliced along many dimensions, such as geography, industry sector, demographics, etc. into a very large collection of granular submarkets, each of which has its own variation in employment. Naturally, this analysis proceeds in two steps: 1) The summarization step: Identify a small set of submarkets which account for a majority of the variation in overall unemployment. 2) Design fixes for negative trends. This second step is most often manually intensive and case specific. Therefore, one hopes that the first step narrows focus meaningfully.

It is commonly observed that hierarchical data lends itself naturally to summarization (cf. OLAP [13]). For instance, the geography dimension in the above example has a natural hierarchy: metros, states, countries, and so on. If all the 
metros in a state have similar unemployment trends, it is more concise to report the state slice as an output of the summary rather than each metro separately. Industry sectors and demographics also have similar hierarchical representations. Organizations are similarly structured hierarchically. The hierarchies aid the allocation, coordination and supervision of tasks that are intended to improve organizational metrics (cf. Organizational Theory [23]). Just as hierarchies in the data inform step 1 from Example 1.1, hierarchies in the organization help effective delegation required to accomplish step 2. For instance, many companies have separate hierarchies for Sales, Finance and Product functions. Data scientists can help analyze performance metrics and assign responsibilities for fixes to the right substructure of the right functional hierarchy.

When determining the submarkets that drive a metric change in step 1, it is important to avoid "double-counting", which can happen if the resulting submarkets are not independent. This is a problem, since it is possible that the same drop in employment is visible in several overlapping slices of data. For instance, a regional slice (e.g. California), and an industry sector (e.g. construction), may both account for the same underlying change in unemployment. Double-counting causes several conceptual problems. It might prevent responsibilities for a fix from being clearly assigned, it might lead to duplicated efforts from two different parts of an organization, or it might lead to the illusion of greater progress than was actually made. For instance, if the construction division and the California division both work on the same issue, or both take credit for a fix, then this credit assignment does not add up. We will therefore insist that the list of submarkets from step 1 are non-overlapping.

Informal problem statement: Identify a small list of non-overlapping sub-segments that account for the majority of a variation in a key metric, where the space of candidate sub-segments is determined by a few, hierarchical dimensions.

Our contributions: Our problem formulation borrows from those of Fagin et al [14] and Sarawagi [26], both of which independently introduce versions of the informal problem statement above. Fagin et al [14] and Sarawagi [26] used lattices to model the hierarchical data. Unfortunately, this leads to the combination of strong hardness results and algorithms that work only for very restricted classes of hierarchies (essentially a single tree).

The key contribution of this paper is to model hierarchical data as a product of trees, which is less general than a lattice. This allows us to circumvent their hardness results. We identify an interesting dynamic-programming algorithm called Cascading Analysts. This algorithm has good performance and runtime, both in a worst-case and an empirical sense. The algorithm is also natural - it mimics the operation of a hierarchical organization of analysts.

Brief contrast with other work: As we discuss in Section 8 in greater detail, there are several formulations of tools and optimization problems that leverage hierarchies within the data. Most of these formalizations are designed to identify and summarize interesting patterns in the data. These tools are usually interactive and require human input to qualify interestingness. This in turn requires the user to have the sophistication of a data analyst. The tools are also designed to be able to handle large amounts of data.

In contrast, we work with a much less general problem, namely to explain the change in a key metric. We seek solutions that does not require any manual intervention. This allows us to serve decision makers that are not necessarily sophisticated in data analysis. Some examples of data-driven decision makers not necessarily sophisticated in data analysis are website developers who want to reason about their traffic, or advertisers who want to reason about their spend (see Section 7). Further, the requirement of coming up with a conclusive answer (without any further interactivity), makes the problem interesting even when the data is not "big", i.e., even when the size of the data is "just" a few megabytes. A decision-maker cannot easily consume far more than ten to twenty lines of output. So even when the number of input segments is a few hundred thousand, there is significant value to the summarization achieved by our algorithm.

\section{PROBLEM STATEMENT}

With the motivation from the previous section, we are ready to state our model and problem formally.

\subsection{Definitions}

We consider multi-dimensional datasets where each dimension can be modeled as a rooted tree. In a single (rooted) tree $T$, we say that two tree-nodes $p$ and $q$ overlap if either $p=q$ or they share an ancestor-descendant relationship, otherwise they are called non-overlapping.

We extend this definition to a cartesian product of trees. For a product of trees $P=T_{1} \times T_{2} \times \cdots \times T_{d}$, we say that two nodes $p=\left(p_{1}, \ldots, p_{d}\right)$ and $q=\left(q_{1}, \ldots, q_{d}\right)$ overlap iff for every dimension $i$, the tree-nodes $p_{i}$ and $q_{i}$ overlap. Consequently, if a pair of nodes does not overlap, then there exists a (possibly non-unique) dimension $i$ such that the tree-nodes $p_{i}$ and $q_{i}$ do not overlap, and we say that nodes $p$ and $q$ do not overlap along dimension $i$.

A node $p=\left(p_{1}, \ldots, p_{d}\right)$ is in the subspace of a node $q=\left(q_{1}, \ldots, q_{d}\right)$ if for every $i, p_{i}$ is either a descendant of $q_{i}$ in the tree $T_{i}$, or $p_{i}=q_{i}$. We define $S u b(v)$ to be the set of nodes that are in the subspace of $v$. We denote the root of the product of the trees as $r=\left(r_{1}, \ldots r_{d}\right)$, where each $r_{i}$ is the root of $T_{i}$.

Finally, a set $S$ of nodes is overlap-free if no two nodes in $S$ overlap.

Example 2.1. Figure 1 depicts the cartesian product of two trees $T_{1}$ and $T_{2}$, each of depth 2 . Tree $T_{i}$ has root $r_{i}$ and left and right children $a_{i}$ and $b_{i}$. Solid lines depict edges between parents and children along tree $T_{2}$, and dotted lines depict the same relationship along tree $T_{1}$. As examples of our definitions, nodes $\left(r_{1}, b_{2}\right)$ and $\left(r_{1}, a_{2}\right)$ do not overlap, but the pair $\left(r_{1}, b_{2}\right)$ and $\left(a_{1}, r_{2}\right)$ does. 


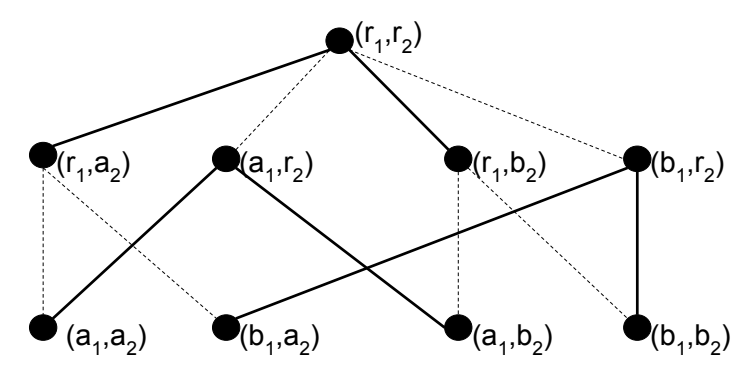

Figure 1: The cartesian product of two trees $T_{1}$ and $T_{2}$, each of depth 2. Each node is a pair of tree nodes. The solid lines are edges along $T_{2}$ and the dotted lines are edges along $T_{1}$.

\subsection{Formal Problem Statement}

With these definitions we can now formally state our problem.

\section{SUMMARIZE}

Input: Trees $T_{1}, T_{2}, \ldots, T_{d}$ with the set of tree-nodes $V:=$ $T_{1} \times T_{2} \times \cdots \times T_{d}$, a non-negative weight function $w: V \rightarrow \mathbb{R}^{+}$, and a maximum output size $k$.

Output: Subset $S \subseteq V$, such that $|S| \leq k, S$ is overlap-free, and $w(S):=\sum_{s \in S} w(s)$ is maximal under these restrictions.

The main parameters of this problem are the number of dimensions $d$, and $n:=|V|$, the size of the input set. We express hardness and runtime results in terms of these two variables.

\subsection{Modeling Metric Changes}

Let us briefly discuss how our problem definition relates to the summarization of metric changes. Assume that we are comparing metrics for two points in time, and that the value of the metric is defined for every leaf node in Figure 1. Via aggregation, this defines the metric values for every internal node as well: The metric value of a parent for one point in time is simply the sum of the metric values of the children.

Let us define the weight function $w$ to be the absolute value of the difference in the metric values for the two time points. (We discuss refinements to this weight function in Section 7.1.)

Why does this weight function result in effective summarization? Consider two patterns of metric changes.

(1) Two children of some node change their metrics in the same direction. Then the node's weight is the sum of children's weights.

(2) Two children of some node change their metrics in different directions. Then the node's weight is smaller in magnitude than one of the children, possibly both.

In the first case, it is better to pick the node rather than the children. In the second case, it is better to pick one or both children rather than the parent. Further, notice that nodes may have multiple parents in different dimensions. So a given input may exhibit both patterns simultaneously, and then it is advantageous to summarize along certain dimensions.

To make this concrete, consider the topology in Figure 1. Suppose that $k=2$ in the above definition. First let us suppose that $\left(a_{1}, a_{2}\right)$ and $\left(b_{1}, a_{2}\right)$ both go up by some quantity $x$, while $\left(a_{1}, b_{2}\right)$ and $\left(b_{1}, b_{2}\right)$ fall by the same quantity $x$. In essence, the change is along dimension 2 .

Notice that the pair $\left(a_{1}, a_{2}\right)$ and $\left(b_{1}, a_{2}\right)$ and the pair $\left(a_{1}, b_{2}\right)$ and $\left(b_{1}, b_{2}\right)$ follow pattern 1 from above. Whereas the pair $\left(a_{1}, a_{2}\right)$ and $\left(a_{1}, b_{2}\right)$ and the pair $\left(b_{1}, a_{2}\right)$ and $\left(b_{1}, b_{2}\right)$ follow pattern 2. Computing the weights shows us that the optimal solution is the overlap-free set consisting of the nodes $\left(r_{1}, a_{2}\right)$ and $\left(r_{1}, b_{2}\right)$, reflecting the change along dimension 2 . Each of these nodes has a weight of $2 x$. The other two internal nodes and the root all have a weight of 0 .

\section{THE CASCADING ANALYSTS ALGORITHM}

As we will discuss in Section 5, Summarize cannot be solved optimally in polynomial time unless $\mathrm{P}=\mathrm{NP}$. Therefore we will now attempt to identify a good approximation algorithm for Summarize. In this section, we describe the "cascading analysts" algorithm that achieves that goal.

\subsection{Conflicts}

Our algorithm will achieve an optimal solution for a more restricted version of SUMMARIZE, namely where the solution is additionally required to be conflict-free.

The presence of a conflict prevents a set of nodes from being recursively subdivided one dimension at a time, even though the set of nodes is possibly overlap-free. This definition and the example that follows elaborate.

Definition 3.1. A conflict is a set $C \subseteq V$ such that for every dimension $i$ there is a $\left(c_{1}, \ldots, c_{d}\right) \in C$ such that for all $\left(x_{1}, \ldots, x_{d}\right) \in C, x_{i}$ is a descendant of $c_{i}$, or $x_{i}=c_{i}$.

A conflict can be overlap-free. Here is the simplest example of a conflict that is also overlap-free.

Example 3.2. Consider three trees $T_{1}, T_{2}, T_{3}$, each of height two, and each with two leaves. Tree $T_{i}$ has root $r_{i}$ and left and right children $a_{i}$ and $b_{i}$ respectively. The conflict is defined by the set of nodes consisting of $\left(r_{1}, b_{2}, a_{3}\right),\left(a_{1}, r_{2}, b_{3}\right)$ and $\left(b_{1}, a_{2}, r_{3}\right)$.

Conflicts play a central role in both the positive and the negative results in our paper. For instance, our algorithm will find the optimal conflict-free solution, i.e., no subset of the nodes output by our algorithm contain a conflict. But it will only be approximate for Summarize: given the input in Example 3.2, our algorithm will only output two of the three nodes, even though the three nodes do not overlap. But we will show that the SummaRIzE problem is NP-hard even over problem instances of three trees of height two like the one in Example 3.2, except with many more leaves. On the other hand, the optimal conflict-free solution can be found by recursively subdividing the product space of trees as we 


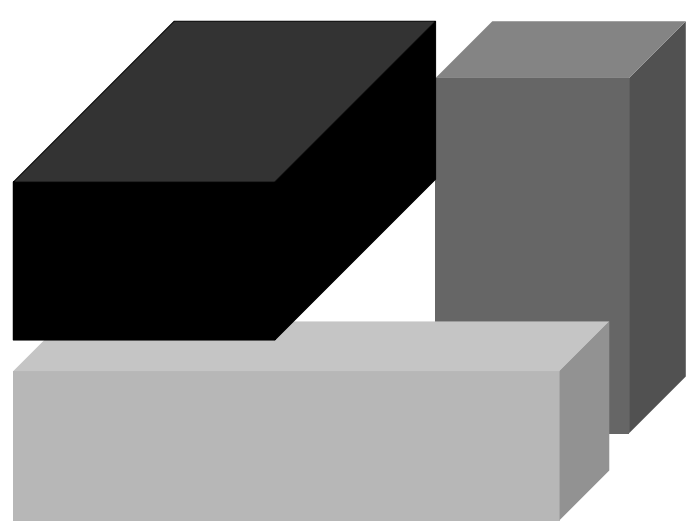

Figure 2: Geometric representation of the conflict in Example 3.2

will show next. Each substep of our algorithm resembles a standard drill-down that an analyst would perform.

The more visually inclined may benefit from a geometric view of a conflict. Figure 2 depicts the conflict in Example 3.2. The three trees in Example 3.2 correspond to the three dimensions in the figure. The three nodes correspond to the three cuboids in the figure. Note that for every dimension, there is a cuboid that spans it completely, i.e., every other cuboid "overlaps" with it in this dimension, or in the words of the the definition of a conflict, is a descendant of this cuboid in this dimension. This overlap prevents us from passing an axis aligned cutting plane through the space that would separate the cuboids without cutting at least one of them. This is the source of the approximation in our algorithm. Our algorithm will recursively perform axis aligned cuts and lose some of the nodes (cuboids) in the process. But as our hardness results in Section 5 show, these conflicts are exactly what make the problem inherently hard, so some of this loss is inevitable.

\subsection{Algorithm}

For each node $v$ in the product of trees $P$, our algorithm computes subsets $S(v, 0), S(v, 1), . ., S(v, k)$ such that for all $j \in\{0, \ldots, k\}: S(v, j) \subseteq S u b(v),|S(v, j)| \leq j, S(v, j)$ is a conflict-free set, and $S(v, j)$ is a maximum weight set satisfying these constraints. The set $S(r, k)$ is the output of our algorithm, where we let $r:=\left(r_{1}, r_{2}, \ldots, r_{d}\right)$ is the root of the product space. The sets $S(v, j)$ are computed bottom-up on the tree product space $P:=T_{1} \times T_{2} \times \cdots \times T_{d}$ via dynamic programming. ${ }^{1}$ These $S(v, j)$, i.e., the solution of a certain cardinality for the tree rooted at $v$, are the subproblems of the dynamic program.

1. Base case: If $v$ is a leaf, i.e. $\operatorname{Sub}(v)=\{v\}$, then we assign the sets in the "obvious" way: $S(v, 0):=\emptyset$, and

\footnotetext{
${ }^{1}$ This dynamic program can either be implemented on a single machine, or distributed using MapReduce.
}

$S(v, j):=\{v\}$ for $j \geq 1$. Recall that the weights are nonnegative, and therefore these settings are optimal.

2. Recursive step: If $S u b(v) \neq\{v\}$, we proceed as follows. We let $S(v, 0):=\emptyset$. For $j \in\{1, \ldots, k\}$, we set $S(v, j)$ as the maximum weight solution among these possibilities:

- The singleton set $\{v\}$.

- The $d$ or fewer solutions $S_{i}(v, j)$ that stem from repeating steps $2 \mathrm{a}$ and $2 \mathrm{~b}$ below for those dimensions $i$ along which $v$ has children, i.e., $v_{i}$ is not a leaf of $T_{i}$.

2a. Breakdown along a dimension $i$ : Let $C_{i}(v)$ be the set of children of $v$ in dimension $i$. So if $v=\left(v_{1}, v_{2}, \ldots, v_{d}\right)$, then:

$C_{i}(v)=\left\{\left(v_{1}, \ldots, v_{i-1}, c, v_{i+1}, \ldots, v_{d}\right) \mid c\right.$ is child of $v_{i}$ in $\left.T_{i}\right\}$

(This is the typical breakdown of the space that an "analyst" interested in dimension $i$ would consider. The algorithm performs a recursive sequence of these, hence the name "cascading analysts".)

We let the maximal solution $S_{i}(v, j)$ along dimension $i$ be the largest weight union of sets of its children $S\left(c_{\ell}, j_{\ell}\right)$ where $c_{\ell} \in C_{i}(v)$ (all $c_{\ell}$ distinct) and $\sum j_{\ell} \leq j$. Note that the number of sets $S\left(c_{\ell}, j_{\ell}\right)$ can be anything from 0 to $j$. This step can be accomplished using a simple dynamic program (not to be confused by the dynamic program over the tree structure) over the children. Here are the details:

2b. Combining child solutions: This simple dynamic program orders the children in $C_{i}(v)$ in a sequence. Let $C_{i}^{m}(v)$ be the first $m$ nodes in $C_{i}(v)$ and $c^{m}$ be the $m$-th child in this sequence. Mirroring the definition of $S(v, j)$, let $S\left(C_{i}^{m}(v), j\right)$ be the optimal conflict-free solution of cardinality at most $j$ in the subspace of the first $m$ children of node $v$. The base case is when $m=1$; here we set $S\left(C_{i}^{m}(v), j\right)=S\left(c^{1}, j\right)$. The recursive step is that we compute $S\left(C_{i}^{m}(v), j\right)$ by selecting the best union of $S\left(C_{i}^{m-1}(v), p\right)$ and $S\left(c^{m}, q\right)$, where $p+q \leq j$. Then, the optimal solution along dimension $i$ is defined by $S\left(C_{i}^{\ell}(v), j\right)$, where $\ell=\left|C_{i}(v)\right|$.

Lemma 3.3. The Cascading Analysts algorithm will output a conflict-free set.

Proof. We prove this claim bottom-up, mirroring the structure of the algorithm. For the base case, when $S u b(v)=$ $\{v\}, S(v, j)$ is either an empty set or a singleton set; both are conflict-free. When $S u b(v) \neq\{v\}$, if $S(v, j)=\{v\}$ then it is conflict-free by itself. Otherwise, there is a dimension $i$ such that $S(v, j)$ is the union of sets each of which is contained within the subspace of a child $c \in C_{i}(v)$.

Suppose that there is a conflict $Q$ in the union. Clearly $Q$ cannot be contained entirely with the subspace of any child, because inductively, these sets are each conflict-free. So $Q$ must span the subspace of at least two distinct children. But then $Q$ cannot be conflict-free because there is no node $q \in Q$ that is an ancestor along dimension $i$ to nodes in the subspace of both these children, violating the condition from the definition of conflicts. So we have a contradiction. 
Lemma 3.4. The Cascading Analysts algorithm will output a maximum weight overlap-free, conflict-free set.

Proof. We first show that all the $S(v, j)$ are overlap-free, i.e. for all values of $v$ and $j$ (recall that $S(r, k)$ is the output of our algorithm). This can be proved inductively. It is obviously true for leaves of the product space (where $S u b(v)=\{v\}$ ). When combining $S\left(c_{\ell}, j_{\ell}\right)$ from different children $c_{\ell}$ note that for elements of $S u b\left(c_{\ell}\right)$ and $S u b\left(c_{\ell^{\prime}}\right)$ have empty intersection in the dimension that we split on ( $i$ in the above description). Thus, their union is overlap-free.

Let $S_{C}$ be a maximum weight, overlap-free, conflict-free solution for an instance of SUmmarize. We will show that the weight of the output of the Cascading Analysts algorithm is at least $w\left(S_{C}\right)$. Since the Cascading Analysts algorithm outputs a conflict-free set (Lemma 3.3), this proves the lemma.

We show the following by induction: for all $v, j$, the weight of $S(v, j)$ is at least the weight of the maximum weight conflict-free subset of size $j$ of $S u b(v)$. Clearly this is true if $v$ is a leaf, so we just need to consider the induction step.

Suppose $w(S(v, j))<w(C)$, where $|C|=j$ and $C \subseteq$ $S u b(v)$ is a conflict-free set. Since $C$ is conflict-free, there has to be a dimension $d^{\prime}$ such that $\forall w \in C: w_{d^{\prime}} \neq v_{d^{\prime}}$. Let $D$ be the children of $v$ in dimension $d^{\prime}$. Then there are children $c_{1}, \ldots, c_{\ell} \in D$ so that $C=C_{1} \cup \cdots \cup C_{\ell}$ where $C_{i} \subseteq S u b\left(c_{i}\right)$. We know that $w\left(S\left(c_{i},\left|C_{i}\right|\right)\right) \geq w\left(C_{i}\right)$ by induction hypothesis. The combination of these sets will be considered by the algorithm, thus $w(S(v, j)) \geq \sum_{i} w\left(S\left(c_{i},\left|C_{i}\right|\right)\right) \geq w\left(C_{i}\right)=$ $w(C)$, contradicting our assumption that the lemma did not hold.

\subsection{Running Time Analysis}

There are $n$ choices of $v$ and $k$ choices of $j$ for which we need to compute the $S(v, j)$. With standard techniques such as memoization, each of these needs to be touched only once. For a fixed $v$, and fixed $j$, we can combine the child solutions to form $S(v, j)$ by a linear pass over the children (as in step $2 \mathrm{a}$ of the algorithm in Section 3.2). Each step in this pass incorporates an additional child and takes time $O(j)$. Since a child can have at most $d$ parents, the total cost of this step for a single node is $O(d j)$. Noting that $j \leq k$, this gives us a total runtime of $O\left(n d k^{2}\right)$.

REMARK 1. Note that the size of the input grows multiplicatively in the number of trees. For example, if each tree has size 10 , then $|V|$ is $10^{d}$. Even reading the input becomes impractical for $d>10$. Fortunately for us, there are compelling practical applications where $d$ is fairly small, i.e. $d \leq 5$. This is true of our applications in Section 7.

\section{PERFORMANCE GUARANTEE}

We first show that our algorithm is optimal for two dimensions, followed by an approximation guarantee of $\left(\left\lceil\log _{2}(n+\right.\right.$ 1) $)^{d-2}$ for the case of three or more dimensions.

TheOrem 2. The Cascading Analysts algorithm solves Summarize optimally when $d=2$.
Proof. We show that when $d=2$, every overlap-free solution is also conflict-free. With Lemma 3.4, this concludes the proof.

All we have to argue is that if a set of nodes $C$ constitutes a conflict, then it also contains an overlap. If $C$ is a conflict, then there are two nodes $x, y \in C, x=\left(x_{1}, x_{2}\right), y=\left(y_{1}, y_{2}\right)$, such that for all $\left(c_{1}, c_{2}\right) \in C, x_{1}$ is an ancestor of $c_{1}$ and $y_{2}$ is an ancestor of $c_{2}$. If $x=y$ then this node overlaps with all other nodes in $C$, completing the proof. If $x \neq y$, then (as just stated) $x_{1}$ is an ancestor of $y_{1}$ and $y_{2}$ is an ancestor of $x_{2}$. Therefore $x$ and $y$ overlap, completing the proof.

Theorem 3. For $d \geq 2$, let $d$ trees $T_{1}, \ldots, T_{d}$ have sizes $n_{1}, \ldots, n_{d}$ respectively. The Cascading Analysts algorithm is $a\left(\left\lceil\log _{2}(m+1)\right\rceil\right)^{d-2}$-approximation algorithm for such an instance of the SUMMARIZE problem, where $m=\max _{i} n_{i}$.

Alternately, The Cascading Analysts algorithm is a $\left(\left\lceil\log _{2}(n+\right.\right.$ 1)ך) $)^{d-2}$-approximation algorithm.

Proof. The second theorem statement is easily implied by the first because $m \leq n$, so we now prove the first statement. Our proof is by induction over $d$. The base case for $d=2$ follows from Theorem 2, so for the following assume $d>2$.

Given a tree, let an (ordered) path be a sequence of nodes $v_{1}, \ldots, v_{h}$ in the tree where $v_{i+1}$ is a child of $v_{i}$ for each $i$. We say that two paths $p_{1}, p_{2}$ overlap if some node $v_{1} \in p_{1}$ overlaps with some node $v_{2} \in p_{2}$. The following combinatorial lemma is fundamental to our proof.

Lemma 4.1. For every rooted tree with $\ell$ leaves, there exists a partition of its nodes into $\left\lceil\log _{2}(\ell+1)\right\rceil$ groups, such that each group is a set of paths, and no two paths in a group overlap.

We defer the proof of Lemma 4.1, and first use it to finish the proof of Theorem 3 .

For a Summarize instance $P$, let $O p t(P)$ denote its optimal solution weight. Let $\beta:=\left\lceil\log _{2}(m+1)\right\rceil$. Using Lemma 4.1, we can decompose $T_{1}$ into disjoint groups $T=G_{1} \cup \cdots \cup G_{g}$, where $g \leq\left\lceil\log _{2}\left(n_{1}+1\right)\right\rceil \leq \beta$.

Let $P_{G_{i}}$ to denote the Summarize problem restricted to $G_{i} \times T_{2} \times \cdots \times T_{d}$. Then we have $\sum_{i} \operatorname{Opt}\left(P_{G_{i}}\right) \geq \operatorname{Opt}(P)$. Wlog assume that $O p t\left(P_{G_{1}}\right)$ has the largest weight among the subproblems, and therefore $\operatorname{Opt}\left(P_{G_{1}}\right) \geq \operatorname{Opt}(P) / g \geq$ $\operatorname{Opt}(P) / \beta$.

Recall that $G_{1}$ is a set of non-overlapping paths. For each such path $p$ in $G_{1}$, consider the Summarize problem $P_{p}$ over $p \times T_{2} \times \cdots \times T_{d}$. Then we have $\sum_{p \in G_{1}} \operatorname{Opt}\left(P_{p}\right) \geq \operatorname{Opt}\left(P_{G_{1}}\right)$.

We remove the first dimension from $P_{p}$ to form a problem $P_{p}^{\prime}$ over $T_{2} \times \cdots \times T_{d}$, by setting $w^{\prime}\left(t_{2}, \ldots, t_{d}\right)$ to be $\max _{v \in p} w\left(v, t_{2}, \ldots, t_{d}\right)$ for all $\left(t_{2}, \ldots, t_{d}\right) \in T_{2} \times \cdots \times T_{d}$. Note that $\operatorname{Opt}\left(P_{p}^{\prime}\right)=\operatorname{Opt}\left(P_{p}\right)$, and a conflict-free solution for $P_{p}^{\prime}$ can be mapped back to a conflict-free solution for $P_{p}$ with the same weight, by replacing each $\left(t_{2}, \ldots, t_{d}\right)$ by $\left(v, t_{2}, \ldots, t_{d}\right)$ where $v=\operatorname{argmax}_{v \in p} w\left(v, t_{2}, \ldots, t_{d}\right)$.

Note that $P_{p}^{\prime}$ has $d-1$ dimensions. By inductive hypothesis, it has a conflict-free solution $S_{p}^{\prime}$ with $w^{\prime}\left(S_{p}^{\prime}\right) \geq O p t\left(P_{p}^{\prime}\right) / \beta^{d-3}$. 
The corresponding conflict-free solution $S_{p}$ for $P_{p}$ also satisfies $w\left(S_{p}\right) \geq \operatorname{Opt}\left(P_{p}\right) / \beta^{d-3}$. Since the $p$ in $G_{1}$ are nonoverlapping, the union of solutions $S:=\cup_{p} S_{p}$ must be a conflict-free solution for $P_{G_{1}}$.

Combining our insights leads to $w(S)=\sum_{p} w\left(S_{p}\right) \geq$ $\sum_{p} \operatorname{Opt}\left(P_{p}\right) / \beta^{d-3} \geq \operatorname{Opt}\left(P_{G_{1}}\right) / \beta^{d-3} \geq \operatorname{Opt}(P) /\left(\beta \beta^{d-3}\right)=$ $\operatorname{Opt}(P) / \beta^{d-2}$.

It remains to show Lemma 4.1. We will prove the following slightly stronger generalization to forests instead. Forests are sets of rooted trees, and nodes in different trees in a forest are considered to be not overlapping.

LEMMA 4.2. For every forest with $\ell$ leaves, there exists a partition of its nodes into exactly $\left\lceil\log _{2}(\ell+1)\right\rceil$ groups, such that each group consists of a set of paths, and no two paths in a group overlap.

Proof. We prove Lemma 4.2 by induction on $\ell$. Given a forest, let $v_{1}, \ldots, v_{\ell}$ be a preorder traversal ordering of its leaves. Let $m:=\lceil\ell / 2\rceil$ be the index of the middle leaf, and let $p$ be the path from $v_{m}$ all the way to its root. If $p$ contains all nodes in the forest, we are done. Otherwise consider the forest over $v_{1}, \ldots, v_{m-1}$, and the forest over $v_{m+1}, \ldots, v_{\ell}$ respectively. The two forests are separated by the path $p$. In particular, no node in the first forest overlaps with any node in the second forest. We inductively apply the lemma to the two (smaller) forests respectively, and obtain $\left\lceil\log _{2}(m-1+1)\right\rceil$ groups for the first forest, and $\left\lceil\log _{2}(\ell-m+1)\right\rceil$ groups for the second forest, both of which have size at most $\left\lceil\log _{2}(\ell+1)\right\rceil-1$. No group from the first forest overlaps with any group from the second forest. Hence we can set $G_{i}$ to be the union of the $i$-th group for the first forest with the $i$-th group for the second forest, for $i=1, \ldots,\left\lceil\log _{2}(\ell+1)\right\rceil-1$, and no two paths in $G_{i}$ overlap. Finally, we finish our construction by setting $G_{\left\lceil\log _{2}(\ell+1)\right\rceil}:=\{p\}$.

REMARK 4. An alternative construction for Lemma 4.2 but with a looser bound is by an induction over the height of the forest, where at each step, we take one root-to-leaf path from each root of the forest, to form a group, and then proceed with the rest of the forest. Each such step reduces the height of the forest by 1, and we end up having as many groups as the height of the forest. In practical applications, the depths of the hierarchies are usually bounded by a small constant such as 3, so that this construction gives a better approximation bound.

REMARK 5. In our applications, the worst-case approximation ratio is usually a small constant $(2-4)$. These applications have at most two large dimensions, which do not contribute to the approximation ratio via the proof of Theorem 3; notice that the proof allows to leave out any two dimensions from the bound, and we may as well leave out the two dimensions that correspond to the largest trees. Further, the other dimensions all have height at most 2. So by Remark 4, we get an approximation factor that is $2^{d-2}$, where $d$ is the number of dimensions. For instance, in the AdWords

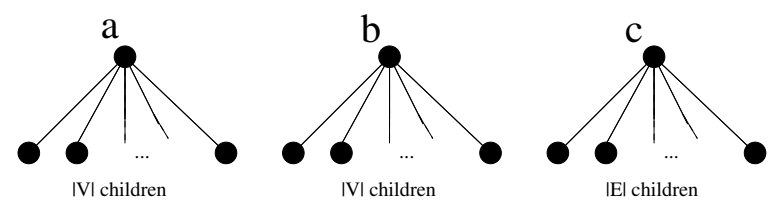

Figure 3: A Summarize problem instance with three trees of height two.

use-case, we get an approximation ratio of 2 . In practice, the approximation could be even better due to the absence of conflicts. We discuss this in Remark 7.

\section{HARDNESS AND LOWER BOUNDS}

\subsection{Hardness results}

We have seen that our algorithm solves SUMmARIzE exactly for $d \leq 2$, and provided approximation guarantees for $d \geq 3$. The following theorem shows that an exact solution even for $d=3$ is likely infeasible.

\section{Theorem 6. Summarize is NP-hard for $d=3$.}

Proof. We show this by reduction from (directed) MAXIMUM IndePENDENT SET (MIS). Given a directed graph $(V, E)$ (an instance of MIS), we construct an instance of SUMmARIZE with $d=3$ as follows. Let us call the three trees $A, B, C$ with roots $a, b, c$, respectively. All the trees have height 2 . In trees $A$ and $B$ we have one child per vertex $v \in V$, called $a_{v}$ and $b_{v}$, respectively. In the third tree, we have one child per edge $(v, w) \in E$, called $c_{v, w}$ (see Figure 3).

The weight function $w$ has non-zero weight on the following nodes:

- node $N_{v}:=\left(a_{v}, b_{v}, c\right)$ has weight 1 for every $v \in V$, and

- nodes $N_{v, w}:=\left(a_{v}, b, c_{v, w}\right)$ and $N_{v, w}^{\prime}:=\left(a, b_{w}, c_{v, w}\right)$ have weight $\beta:=1+\varepsilon$ for every $(v, w) \in E$.

We set $k=\infty$, so that any overlap-free set $S$ is a valid solution. Note that the reduction is polynomial-time; the number of nodes in the SUMmaRIzE instance is $O\left(|E| \cdot|V|^{2}\right)$.

We claim that there is a solution to MIS of size $m$ if and only if there is a solution $S$ to this instance of SUMMARIZE with $w(S) \geq m+\beta|E|$. This implies that Summarize is NP-hard.

" $\Rightarrow$ ": Let $V^{\prime} \subseteq V$ be an independent set with $\left|V^{\prime}\right|=m$. Let $S$ be the union of the sets $S_{1}:=\left\{N_{v} \mid v \in V^{\prime}\right\}, S_{2}:=$ $\left\{N_{v, w} \mid v \notin V^{\prime}\right\}, S_{3}:=\left\{N_{v, w}^{\prime} \mid v \in V^{\prime} \wedge w \notin V^{\prime}\right\}$.

Since for all edges $(v, w)$ either $v$ or $w$ is not in $V^{\prime}$, we have that either $N_{v, w} \in S_{2}$ or $N_{v, w}^{\prime} \in S_{3}$, and thus $\left|S_{2} \cup S_{3}\right|=|E|$. Therefore, we have $w(S)=m+\beta|E|$. It remains to show that $S$ is overlap-free. There are no overlaps in $S_{2} \cup S_{3}$, since no two elements overlap in the third dimension $\left(c_{v, w}\right)$, neither are there overlaps in $S_{1} \cup S_{2}$, since elements differ in the first dimension, and in $S_{1} \cup S_{3}$, since elements differ in the second dimension.

" $\Leftarrow$ ": Given a solution $S$ to the Summarize problem with $w(S) \geq m+\beta|E|$, we need to construct an independent set of 
size at least $m$. For each edge $(v, w), S$ can contain at most one of $N_{v, w}$ and $N_{v, w}^{\prime}$, since the two nodes overlap. However, wlog we can assume that $S$ contains exactly one of them: It is not hard to see that if $S$ contains neither, and adding one of them would create an overlap, then $S$ has to contain either $N_{v}$ or $N_{w}$. However replacing e.g. $N_{v}$ by $N_{v, w}$ will increase the weight of $S$ by $\beta-1=\varepsilon$. Note that this also implies that for any edge $(v, w), N_{v}$ and $N_{w}$ cannot both be in $S$.

So wlog, $S$ contains exactly one of $N_{v, w}$ and $N_{v, w}^{\prime}$ for each edge $(v, w)$. Since $w(S) \geq m+\beta|E|$, at least $m$ of the weight comes from nodes $N_{v}$. Thus, the set $V^{\prime}:=\left\{v \mid N_{v} \in S\right\}$ satisfies $\left|V^{\prime}\right| \geq m$. As we observed before, for each edge $(v, w)$, not both $v$ and $w$ can be in $V^{\prime}$, thus it is an independent set of the desired size.

Remark 7 (The Computational Lens on Summarize). As stated by Lemma 3.4, SUMMARIZE identifies the optimal conflict-free solution. One potential concern is that conflicts are just an artifact of our algorithm, and that had we been more imaginative, we could have come up with algorithms

Note the fundamental role played by conflicts in the proofs of Theorem 6. The simple conflict in Example 3.2 underlies the constructions in the proof.

\subsection{Lower bounds for the algorithm}

We now construct "hard" input instances for SUMmARIzE for which our algorithm outputs a solution that has $(2 / 3)^{d / 3}$ of the weight of the optimal solution, when $d$ is a multiple of 3 . It follows that our algorithm is at best a $(3 / 2)^{\lfloor d / 3\rfloor}>1.144^{d-2}$ approximation algorithm. Our strategy will be to construct an instance with lots of conflicts.

THEOREM 8. For every integer $m \geq 1$, there is an instance of Summarize with $d=3 m$ dimensions that has an overlapfree solution with weight $3^{m}$, while the optimal conflict-free solution has weight $2^{m}$.

Proof. Note that for $m=1$, such a problem instance is given by the conflict in Example 3.2. We obtain the general case by raising this example to the " $m$-th power", as follows.

Our instance of SUMMARIZE has $d=3 m$ dimensions, where every dimension has a tree with root $r_{i}$ and two children $a_{i}$ and $b_{i}$. We group the dimensions into sets of size three, and define for $i \in\{1, \ldots, m\}$ :

$S_{i}:=\left\{\left(a_{3 i-2}, b_{3 i-1}, r_{3 i}\right),\left(b_{3 i-2}, r_{3 i-1}, a_{3 i}\right),\left(r_{3 i-2}, a_{3 i-1}, b_{3 i}\right)\right\}$

Note that these are copies of Example 3.2 restricted to dimensions $3 i-2,3 i-1,3 i$. The weight function $w$ is 1 for all nodes in $S:=S_{1} \times S_{2} \times \cdots \times S_{m}$; all other nodes have weight zero. We set $k=\infty$.

By construction, $S$ has $3^{m}$ elements, and therefore a total weight of $3^{m}$. For the first claim, we now show that $S$ is overlap-free. Consider two different elements of $S$. Clearly, they must differ on some factor $S_{i}$. But by definition of $S_{i}$ that means that they are disjoint.

For the second claim, we now inductively prove that every conflict-free solution of this problem instance has a weight of at most $2^{m}$. For $m=1$, the claim follows from Example 3.2.
For $m \geq 2$, let $T \subseteq S$ be a conflict-free solution. We can assume $|T|>1$, since otherwise $T$ clearly is of size less than $2^{m}$.

Since $T$ is conflict-free, there must be a dimension $i$ such that $x_{i} \in\left\{a_{i}, b_{i}\right\}$ for all $x \in T$ (as per the definition of a conflict). By symmetry of our construction, we can wlog assume $i=d$. Then $T$ can be decomposed as a disjoint union $T=T_{a} \cup T_{b}$, where $T_{a}=T \cap\left\{x \mid x_{d}=a_{d}\right\}$, and $T_{b}=T \cap\left\{x \mid x_{d}=b_{d}\right\}$. By construction of $S$, the nodes in $T_{a}$ have the same values in factor $S_{m}$. Removing the last three dimensions from $T_{a}$, we obtain a set of nodes in $S_{1} \times \cdots \times S_{m-1}$ with the same cardinality. This set is also conflict-free (otherwise $T_{a}$ would contain a conflict), and forms a solution to the instance of size $m-1$. By induction, $T_{a}$ can have size at most $2^{m-1}$. Similarly, $\left|T_{b}\right| \leq 2^{m-1}$, and therefore $|T|=\left|T_{a}\right|+\left|T_{b}\right| \leq 2^{m}$.

REMARK 9 (DENSE INPUT VERSUS SPARSE INPUT). There is a significant difference between our work and Multi-structural Databases [14] in how the input is provided. In [14], the input consists only of the subset $V^{\prime}$ of nodes that have non-zero weight. Let us call this a sparse input. In contrast, we assume that the weights are specified for every node $v \in V$. That is, we assume that the input is dense. We chose this modeling assumption because in practice (i.e. for the applications in Section 7), we found that almost all nodes in $V$ had non-zero weight. Even though our algorithm is described for the dense case, it is straightforward to apply it to the sparse case as well.

Sparseness plays a critical role in the hardness results of [14]. They perform a reduction from independent set (a well-known NP-hard problem). Their reduction can be done in polynomial time only if the input is sparse. In fact, in their reduction, the number of nodes is equal to the number of trees.

These results therefore do not imply that the dense case is also NP-hard. In principle, it is possible that NP-hardness disappears because we 'only' need to be polynomial in the product of the sizes of the trees. Theorem 6 shows that the problem is NP-hard even with dense input. So the hardness is not due to the density of input, but due to the presence of conflicts.

Remark 10 (Comparison to Rectangle Packing). It is instructive to compare SUMMARIZE to the problem of the max-weight packing of axis-aligned hyper-rectangles in a space so that no two rectangles spatially overlap. Nodes in our problem correspond to hyper-rectangles in that setting (cf [7, 9, 19]). That problem is optimally solvable for one dimension and NP-Hard for two more more dimensions. It can be shown that every instance of our problem is an instance of that problem, but not vice versa. This is because a tree cannot model the case where two hyper-rectangles intersect along a certain dimension, but neither contains the other. What Theorems 6 and 2 together show is that the restriction to hierarchical hyper-rectangles now allows positive results for two dimensions and "postpones" the NP-Hardness to three or 
more dimensions. We borrow some proof ideas for Theorem 3 from [7].

\section{EMPIRICAL EVALUATION}

Section 4 presented a worst-case evaluation of our algorithm. In this section, we complement this with an empirical evaluation over an employment data set from the American Community Survey [6].

\subsection{Employment data}

The table we investigate has three dimensions: sex, occupation, and geography. Sex has two values. Occupation is a five level hierarchy of size 126 for years $2005-2009$ and 151 for years $2010-2015$. Geography is organized as a three level hierarchy with 50 states, 9 divisions and 4 regions. We produce summarizations for six comparisons; we compare 2007,2008, 2009 against 2006 and 2011,2013,2015 against 2010. We investigate four different cardinality constraints (values of $k$ ): 10,20,50,100. We use the simple weight function discussed in Section 2: For each node $v, w(v)$ is set to the absolute value of the difference in the employment values for the node between two time periods $\left|t_{v}-l_{v}\right|$, where $l_{v}$ is the employment value for the current time period, and $t_{v}$ is the empoyment value for a past time period.

\subsection{Some Qualitative Results}

We start by manually inspecting the output of Cascading Analysts. Table 1 compares the figures for 2009 against 2006 with a cardinality parameter of 5 . The large drops in construction and production related occupations are likely recession related. In contrast, management and service related occupations saw an increase. Table 2 repeats the same comparison with a larger cardinality parameter (10). Cascading Analysts chooses to use the extra cardinality to provide more detail for sales related occupations. This choice is justified by the fact that one of the subsegments within sales, cashiers, saw a percentage increase of $5 \%$, whereas the other subsegments of sales saw a drop. Next, we compare 2013 against 2010. Notice that both years are post recession, and the output summarizes a very different trend. This time Cascading Analysts chooses to segment the data along sex, and then slice females by occupation; this seems justified by the large variation precentage changes of occupations for females.

\subsection{Baselines}

We compare our algorithm against two baselines. The first baseline is from the prior work of Fagin et al. [14] and Sarawagi [26] on which our model is based. Motivated by computational hardness results, these works proposed an algorithm that coerces the lattice (product of trees) into a single tree by sequentially composing dimensions (intuitively, pasting one dimension below another). Fagin et al. [14] refers to this as a sequential pairwise disjoint collection. There are $d$ ! ways to compose the dimensions into a tree where $d$ is the number of dimensions. Both Fagin et al. [14] and Sarawagi [26] expect the permutation of the dimensions to be input by the user, although it is hard for the user to guess the best permutation in advance. So we will report the performance of our algorithm against the best, worst and average case over these permutations.

We also compare against the optimal solution to the SUMMARIZE problem as computed using an integer program solver. Integer program solvers do produce the optimal solution when the data is sufficiently small. Here is how we construct the integer program: for every internal node $v$, we introduce a $0 / 1$ variable $x_{v}$ to indicate whether $v$ is part of the solution. Then we use $\sum_{v} x_{v} \leq k$ to enforce the constraint on output size, and we use $\sum_{v}$ an ancestor of $l \leq 1$ for every leaf node $l$ to enforce that no two chosen nodes overlap. Such integer programs tend to run for excessively long times for large datasets. We consider them mainly for the purpose of quantifying the loss of approximation.

We do not compare against OLAP tools like [18] that require a human to drive the construction of an explanation. This is not because these tools are inherently inferior, but because the quality of the results will certainly depend on the ability and diligence of the human, and this is hard to model. As emphasized in the introduction, our goal is to completely automate the task of constructing an explanation of metric changes, and we choose our baselines accordingly.

\subsection{Runtime comparison}

We compare the run time of three algorithms: Cascading Analysts, tree composition, and integer program solver. Note that the tree composition algorithm expects the user to specify an ordering of trees. There are six trees available for our dataset. We measure the run time for all the six orderings, and report the mean and sum values. If the ordering is specified, the mean value is a good measurement of run time. If the algorithm searches for the best ordering, the sum value is a good measurement of run time.

We note that the tree composition algorithm is slightly faster than Cascading Analysts if the ordering of tree is specified. That is expected as it involves a simpler dynamic program. Having to try out all six orderings roughly increases the run time six-fold. Integer program solvers are unsurprisingly slower.

One may think that the integer program solver is computationally efficient for our problem despite the NP Hardness result. To test the boundary of integer program solvers, we first synthesized a dataset with three trees, where each tree has depth three, and every node in the tree has degree three, with values are drawn IID from a standard normal distribution. Both Cascading Analysts and integer programs run without timing out for this dataset. When we change one of the trees to have depth four, and our program for generating the integer program takes longer than half-an-hour to terminate. So we are at the boundary of what integer programming can handle (see Table 5 for details). 


\begin{tabular}{llrrr}
\hline Geo Sex & Occupation & 2006 & 2009 & percentage change \\
\hline & - Management, professional, and related occupations & $48.06 \mathrm{M}$ & $50.18 \mathrm{M}$ & \\
& - Construction, extraction, maintenance, and repair occupations & $14.09 \mathrm{M}$ & $12.27 \mathrm{M}$ & -12.9 \\
& - Service occupations & $23.36 \mathrm{M}$ & $25.07 \mathrm{M}$ & 7.3 \\
& - Production, transportation, and material moving occupations & $18.36 \mathrm{M}$ & $16.67 \mathrm{M}$ & -9.2 \\
& - Sales and office occupations & $36.62 \mathrm{M}$ & $35.43 \mathrm{M}$ & \\
\hline
\end{tabular}

Table 1: Comparing U.S. Employment 2009 vs. 2006., cardinality 5. The large drops in construction and production related occupations are likely recession related. In contrast, management and service related occupations saw an increase.

\begin{tabular}{|c|c|c|c|c|c|}
\hline Geo & Sex & Occupation & 2006 & 2009 & percentage change \\
\hline & & — Management, professional, and related occupations & $48.06 \mathrm{M}$ & $50.18 \mathrm{M}$ & 4.4 \\
\hline & & - Construction, extraction, maintenance, and repair occupations & $14.09 \mathrm{M}$ & $12.27 \mathrm{M}$ & -12.9 \\
\hline & & — Service occupations & $23.36 \mathrm{M}$ & $25.07 \mathrm{M}$ & 7.3 \\
\hline & & — Production, transportation, and material moving occupations & $18.36 \mathrm{M}$ & $16.67 \mathrm{M}$ & -9.2 \\
\hline & & - Sales and office occupations - Office and administrative support occupations & $20.4 \mathrm{M}$ & $19.68 \mathrm{M}$ & -3.5 \\
\hline & & — Sales and office occupations — Sales and related occupations - Other sales and related workers including supervisors - ${ }^{*}{ }^{*}$ & $6.04 \mathrm{M}$ & $5.71 \mathrm{M}$ & -5.4 \\
\hline & & — Sales and office occupations — Sales and related occupations — Sales representatives, services, wholesale and manufacturing_ $*$ — & $3.42 \mathrm{M}$ & $3.22 \mathrm{M}$ & -5.7 \\
\hline & & - Sales and office occupations - Sales and related occupations - Cashiers_- *_* & $3.07 \mathrm{M}$ & $3.22 \mathrm{M}$ & 5.0 \\
\hline & & — Sales and office occupations — Sales and related occupations — Retail sales workers except cashiers_ * _ * & $3.69 \mathrm{M}$ & $3.58 \mathrm{M}$ & -2.9 \\
\hline - South & & — Farming, fishing, and forestry occupations & $0.32 \mathrm{M}$ & $0.3 \mathrm{M}$ & -7.6 \\
\hline
\end{tabular}

Table 2: Comparing U.S. Employment 2009 vs. 2006., cardinality 10. This repeats the previous comparison but with a larger cardinality parameter (10). Cascading Analysts chooses to use the extra cardinality to provide more detail for sales related occupations. This choice is justified by the fact that one of the subsegments within sales, cashiers, saw a percentage increase of $5 \%$, whereas the other subsegments of sales saw a drop.

\begin{tabular}{|c|c|c|c|c|c|}
\hline Geo & Sex & Occupation & pre & post & percentage change \\
\hline & - Male & & $72.38 \mathrm{M}$ & $76.15 \mathrm{M}$ & 5.2 \\
\hline & - Female & — Management, business, science, and arts occupations & $26.26 \mathrm{M}$ & $27.52 \mathrm{M}$ & 4.8 \\
\hline & - Female & - Service occupations & $14.22 \mathrm{M}$ & $15.04 \mathrm{M}$ & 5.8 \\
\hline & - Female & - Sales and office occupations - Office and administrative support occupations - Customer service representatives $-*{ }^{*}{ }^{*}$ & $1.51 \mathrm{M}$ & $1.7 \mathrm{M}$ & 12.5 \\
\hline & - Female & — Sales and office occupations - Office and administrative support occupations — Secretaries and administrative assistants_ $*$ _ * & $3.42 \mathrm{M}$ & $3.27 \mathrm{M}$ & -4.3 \\
\hline & - Female & - Sales and office occupations - Office and administrative support occupations - Bookkeeping, accounting, and auditing clerks - * - * & $1.19 \mathrm{M}$ & $1.11 \mathrm{M}$ & -6.3 \\
\hline & - Female & - Sales and office occupations - Office and administrative support occupations - First - line supervisors of office and administrative support workers - * & $0.87 \mathrm{M}$ & $0.8 \mathrm{M}$ & -7.2 \\
\hline & - Female & - Sales and office occupations - Office and administrative support occupations - Information and record clerks except customer service representatives $-*$ - $*$ & $2.24 \mathrm{M}$ & $2.2 \mathrm{M}$ & -1.7 \\
\hline & - Female & - Sales and office occupations - Sales and related occupations & $7.66 \mathrm{M}$ & $7.85 \mathrm{M}$ & 2.4 \\
\hline & - Female & - Production, transportation, and material moving occupations & $3.78 \mathrm{M}$ & $3.92 \mathrm{M}$ & 3.6 \\
\hline
\end{tabular}

Table 3: Comparing U.S. Employment 2013 vs. 2010, cardinality 10. Notice that both years in this comparison are post recession, and the output summarizes a very different trend. This time Cascading Analysts chooses to segment the data along sex, and then slice females by occupation; this seems justified by the large variation precentage changes of occupations for females.

\subsection{Approximation Comparison}

We report the ratio of the optimal solution as computed by the integer program to that of the Cascading Analysts and the tree composition algorithm; this is the approximation achieved by the algorithms. For the tree composition algorithm, there are 3 ! candidate solutions, one per ordering of the dimensions. We report the best, worst, and average over these permutations. Table 6 describes our results. The main take-aways are as follows. Our algorithm produces the optimal result almost every time, and when it does not, it is very close to optimal. The mean of the tree compositions is up to $7 \%$ worse than our algorithm, and the worst ordering is up to $15 \%$ worse than our algorithm. The best of the tree compositions also produces a nearly optimal solution every time. But as we observed in the previous subsection, it is four times slower to compute the tree composition for all permutations of dimensions. Further, the solution yielded by the best permutation will never be better than Cascading Analysts because these solutions are always conflict-free and the Cascading Analysts algorithm produces the best conflict-free solution. Further, in the worst-case, the tree composition algorithm (even with the best permutation) can perform quite badly. We show an example where it only has a $1 /(n-1)^{1 / 4}$ fraction of the weight of Cascading Analysts (see the Appendix A). Finally, Table 7 includes a comparison on a synthetic data set (three trees of depth three, each internal node has a degree three, and data drawn IID from the normal distribution). Now Cascading Analysts is $1-5 \%$ better than even the best tree composition, and $3-11 \%$ better on average.

Remark 11 (Conficts in Practice). Recall that the Cascading Analysts produces the optimal conflict-free solution. The fact that Cascading Analysts algorithm is optimal or very nearly optimal on all the problem instances in our 


\begin{tabular}{|l|r|r|r|r|r|}
\hline pre vs. post & $\mathrm{k}$ & C.A. & tree mean & tree sum & IP time \\
\hline 07 vs. 06 & 10 & 2.102 & 2.424 & 14.545 & 71.228 \\
07 vs. 06 & 20 & 2.291 & 2.787 & 16.721 & 79.150 \\
07 vs. 06 & 50 & 6.166 & 3.216 & 19.295 & 73.627 \\
07 vs. 06 & 100 & 9.181 & 3.793 & 22.757 & 70.277 \\
08 vs. 06 & 10 & 2.056 & 2.666 & 15.995 & 90.672 \\
08 vs. 06 & 20 & 3.685 & 2.763 & 16.576 & 69.240 \\
08 vs. 06 & 50 & 4.259 & 3.225 & 19.352 & 83.793 \\
08 vs. 06 & 100 & 7.465 & 3.856 & 23.134 & 69.654 \\
09 vs. 06 & 10 & 2.984 & 2.109 & 12.656 & 78.868 \\
09 vs. 06 & 20 & 2.585 & 2.201 & 13.205 & 70.543 \\
09 vs. 06 & 50 & 4.925 & 3.234 & 19.406 & 76.650 \\
09 vs. 06 & 100 & 6.985 & 3.789 & 22.733 & 72.358 \\
11 vs. 10 & 10 & 3.455 & 3.279 & 19.672 & 102.092 \\
11 vs. 10 & 20 & 3.820 & 3.497 & 20.983 & 98.701 \\
11 vs. 10 & 50 & 6.176 & 3.920 & 23.522 & 97.705 \\
11 vs. 10 & 100 & 9.244 & 4.705 & 28.227 & 98.145 \\
13 vs. 10 & 10 & 4.058 & 3.290 & 19.741 & 120.054 \\
13 vs. 10 & 20 & 4.621 & 3.458 & 20.749 & 118.622 \\
13 vs. 10 & 50 & 7.163 & 3.986 & 23.914 & 146.397 \\
13 vs. 10 & 100 & 10.789 & 4.408 & 26.446 & 117.223 \\
15 vs. 10 & 10 & 4.059 & 2.748 & 16.485 & 111.192 \\
15 vs. 10 & 20 & 4.035 & 2.776 & 16.657 & 100.918 \\
15 vs. 10 & 50 & 7.199 & 3.514 & 21.083 & 110.777 \\
15 vs. 10 & 100 & 10.971 & 4.143 & 24.855 & 134.285 \\
\hline
\end{tabular}

Table 4: Run time in seconds for Cascading Analysts (C.A.), tree composition algorithm, and integer program solver for different data sets and different summarization cardinalities $(\mathrm{k})$.

\begin{tabular}{|l|r|r|r|}
\hline tree depths & $\mathrm{k}$ & C.A. analyst & IP time \\
\hline $3,3,3$ & 10 & 7.282 & 157.469 \\
$3,3,3$ & 20 & 8.478 & 162.294 \\
$3,3,3$ & 50 & 12.456 & 162.477 \\
$3,3,3$ & 100 & 22.641 & 363.935 \\
$3,3,4$ & 10 & 28.222 & $>1800$ \\
$3,3,4$ & 20 & 26.401 & $>1800$ \\
$3,3,4$ & 50 & 42.177 & $>1800$ \\
$3,3,4$ & 100 & 79.949 & $>1800$ \\
\hline
\end{tabular}

Table 5: Run time in seconds for Cascading Analysts (C.A.), and integer program solver for synthetic datasets.

\begin{tabular}{|l|r|r|r|r|r|}
\hline pre vs. post & $\mathrm{k}$ & C.A. & tree mean & tree min & tree max \\
\hline 07 vs. 06 & 10 & 1.000 & 1.010 & 1.000 & 1.018 \\
07 vs. 06 & 20 & 1.000 & 1.015 & 1.000 & 1.031 \\
07 vs. 06 & 50 & 1.000 & 1.043 & 1.007 & 1.079 \\
07 vs. 06 & 100 & 1.000 & 1.053 & 1.009 & 1.100 \\
08 vs. 06 & 10 & 1.000 & 1.000 & 1.000 & 1.000 \\
08 vs. 06 & 20 & 1.000 & 1.001 & 1.000 & 1.002 \\
08 vs. 06 & 50 & 1.000 & 1.022 & 1.003 & 1.042 \\
08 vs. 06 & 100 & 1.001 & 1.037 & 1.005 & 1.070 \\
09 vs. 06 & 10 & 1.000 & 1.000 & 1.000 & 1.000 \\
09 vs. 06 & 20 & 1.000 & 1.000 & 1.000 & 1.000 \\
09 vs. 06 & 50 & 1.000 & 1.006 & 1.000 & 1.012 \\
09 vs. 06 & 100 & 1.000 & 1.022 & 1.001 & 1.043 \\
11 vs. 10 & 10 & 1.000 & 1.003 & 1.001 & 1.006 \\
11 vs. 10 & 20 & 1.000 & 1.002 & 1.000 & 1.004 \\
11 vs. 10 & 50 & 1.000 & 1.017 & 1.000 & 1.037 \\
11 vs. 10 & 100 & 1.001 & 1.076 & 1.005 & 1.156 \\
13 vs. 10 & 10 & 1.000 & 1.002 & 1.000 & 1.004 \\
13 vs. 10 & 20 & 1.000 & 1.001 & 1.000 & 1.003 \\
13 vs. 10 & 50 & 1.000 & 1.008 & 1.000 & 1.017 \\
13 vs. 10 & 100 & 1.000 & 1.027 & 1.001 & 1.055 \\
15 vs. 10 & 10 & 1.000 & 1.001 & 1.000 & 1.001 \\
15 vs. 10 & 20 & 1.000 & 1.001 & 1.000 & 1.001 \\
15 vs. 10 & 50 & 1.000 & 1.004 & 1.001 & 1.007 \\
15 vs. 10 & 100 & 1.000 & 1.015 & 1.002 & 1.029 \\
\hline
\end{tabular}

Table 6: Approximation ratio for Cascading Analysts and tree composition algorithms for different data sets and different summarization cardinalities (k). A ratio of 1.0 signifies optimality.

employment data analysis shows that the optimal solution in these examples either do not contain conflicts, or do not

\begin{tabular}{|l|r|r|r|r|}
\hline $\mathrm{k}$ & C.A. & tree mean & tree min & tree max \\
\hline 10 & 1.0 & 1.036 & 1.011 & 1.060 \\
20 & 1.0 & 1.08 & 1.012 & 1.125 \\
50 & 1.001 & 1.116 & 1.051 & 1.164 \\
100 & 1.001 & 1.110 & 1.061 & 1.159 \\
\hline
\end{tabular}

Table 7: Approximation ratio for Cascading Analysts and tree composition algorithms for the synthetic dataset with three depth-3 trees, for different cardinality (k). A ratio of 1.0 signifies optimality.

contain large weight conflicts. Our intuition is that in the context of summarizing metric changes, it is likely that conflicts are fairly rare in the optimal solution because for even the simplest one to manifest (as in Example 3.2), there have to be three fluctuations, each from three separate causes, but the causes do not overlap with each other. It would be worthwhile to see if this conjecture holds more generally in practice.

\section{APPLICATIONS OF THE ALGORITHM}

The Cascading Analysts algorithm is fairly general. The key choices when applying the algorithm to a specific context are to pick the metrics and dimensions to apply them over, and a sensible weight function. We have applied the Cascading Analysts algorithm to help advertisers debug their advertising campaigns via a report called the "top movers report" [5] , and to help websites analyze their traffic. within Google Analytics [2].

\subsection{Interesting Weight Functions}

In Section 2, we discussed a very simple weight function used to analyze metric changes. For each node $v, w(v)$ was set to the absolute value of the difference in the metric values for the node between two time periods $\left|t_{v}-l_{v}\right|$, where $l_{v}$ is the metric value for the current time period, and $t_{v}$ is the metric value for a past time period (pre-period). In this section, we present other alternatives that result in different types of summarization.

7.1.1 Modeling Composition Change. If the data displays a generally increasing trend (or a generally decreasing trend), it is possible that almost all the slices are data are generally increasing. So the weight function $w(v)=\left|t_{v}-l_{v}\right|$ essentially becomes $w(v)=t_{v}-l_{v}$, and the root node is a degenerate, optimal solution, because it has at least as much weight as any set of non-overlapping nodes. In practice we may still want to separate low growth slices from high growth ones, because the former could still be improved. A simple option is to compare the mix or the composition of the metric rather than magnitude of the metric, that is

$$
w(v):=\left|\frac{t_{v}}{\sum_{v \in V} t_{v}}-\frac{l_{v}}{\sum_{v \in V} l_{v}}\right|
$$

This way, the output of SUMmARIZE consists of nodes that were a large fraction of the market in one of the two time periods, and a relatively small fraction in the other. 
This technique is also useful in performing Benchmarking [1]. In Benchmarking the goal is to compare the metric for a business against similar businesses. For instance, comparing the traffic of one messaging app against the traffic of another app across dimensions such as location, user age, etc. Here, $t_{v}$ 's correspond to amount of traffic for the protagonist's company/app and $l_{v}$ 's represent traffic for a benchmark app. It is usually the case that one of the businesses/apps is much larger, and therefore it makes more sense to compare the composition of their markets as in Equation 1.

7.1.2 Modeling Absolute Change v/s Relative Change. A slightly different issue is that a large relative change in a metric (say from $\$ 500$ to $\$ 1000$ ) may be more interesting than a small relative change in metric (say from $\$ 10500$ to $\$ 11000)$, because the latter is probably due to usual fluctuations (noise), whereas the former is a real event worth responding to. However, focusing entirely on relative change could produce tiny segments as output (say the metric changes from 1 to 100). In practice, it makes sense to weigh relative changes to some extent. To model this, we apply a standard technique called a Box-Cox transform [10] by setting the weight $w(v):=\frac{l_{v}^{1-m}-t_{v}^{1-m}}{1-m}$. For $m=0$, this reduces to the absolute value of the difference $\left|l_{v}-t_{v}\right|$. When $m \rightarrow 1$, this approaches $\log \left(l_{v}\right)-\log \left(t_{v}\right)$, which models a relative difference. In practice we found it useful to set $m$ in the range $[0.1,0.3]$.

\subsection{Analyzing the Performance of Ad Campaigns}

Google's Adwords [3] platform enables advertisers to run pay-per-click advertising campaigns. There are many factors that affect the performance of a campaign. The advertiser's own actions, an advertiser's competitor's actions, a change in user behavior, seasonal effects, etc. Given that large amounts of money are often at stake, most advertisers monitor and react to changes in their spend very carefully.

A routine task for advertisers is therefore to (1) periodically identify the top moving data-segments, and (2) react to these changes sensibly. We seek to automate (1). Adapting the Cascading Analysts algorithm involves carefully choosing the dimensions and the weight function. We launched our Cascading Analysts algorithm in the adwords "top movers report" in June 2013 [5].

We use three hierarchical dimensions to partition campaign performance. The first is the campaign/group-of-ads/keyword hierarchy. Each campaign can have multiple groups of ads, and a group of ads can have multiple keywords, forming a tree structure. For example, a gift shop can have one campaign for flowers and one campaign for cards. Within the flower campaign, it can have one adgroup for each zip code that the shop serves. A second hierarchical dimension partitions the user by the kind of device they use. The top level split can be Mobile, Desktop, and Tablet, and each of these can further be split by the make and model of the device. And the third dimension is the platform on which the ads were shown, for instance, search, the web, or a social network.

We briefly describe how the weight function is modeled. The metrics of interest are the spend of the advertiser, the number of clicks received by the ads, and the number of views (impressions) received by the ads. We usually compare two time-slices. The weight function is modeled as the BoxCox transformation applied to the values of the metric in the two time-periods (see Section 7.1.2).

\subsection{Understanding Website Traffic}

Google Analytics helps website and app developers understand their users and identify opportunities to improve their website (and similarly for phone apps) [4].

There are many factors that affect the traffic to a website. Changes in user interests, buzz about the website in social media, advertising campaigns that direct traffic to the website, changes to the website that make it more or less engaging, etc. The "Automated Insights" feature of Google Analytics [2] analyzes the traffic data and identifies opportunities or room for improvement. The Cascading Analysts algorithm is used to identify focus-worthy segments of traffic. The feature involves other algorithms and curation to make the insights actionable.

We now discuss the metrics and the dimensions. There are a wide variety of apps and websites, for example by content providers like large newspapers, ecommerce platforms, personal websites or blogs, mobile apps, games, etc. Different dimensions and metrics are important for different businesses. Consequently, Google Analytics has a very large set of dimensions and metrics that it supports. Some metrics include visits to the website, number of users, number of sessions, and a metric called goals whose semantics are user-defined. Some examples of dimensions include the source of the traffic to the website (search engines, social network sites, blogs, direct navigation), medium (was the visit due to an ad, an email, a referral), geographic dimensions (continent, country, city), device related dimensions (as in our advertising example above) etc. The Cascading Analysts algorithm is applied to several coherent groupings of dimensions and metrics. For instance, we may run the algorithm to compare the composition (see Section 7.1.1 of visits in one month versus another, with three dimensions like source, geography and device. (Here we compare compositions rather than the raw metric magnitudes because large seasonal effects could make all the data trend up or down.) This produces several candidate segments that are then turned into insights reports.

\section{RELATED WORK}

\subsection{OLAP/Drill-Down}

There is a large body of literature on OLAP [13]. As discussed in the introduction, there is justified interest in automating data analysis for it. There is work on automating or helping the automation of drill-downs [16, 21, 27, 29, 30]. These attempts to summarize patterns in the data use informationtheoretic approaches rather than explain the difference in 
aggregate metrics. Candan et al [12] propose an extension to OLAP drill-down that takes visualization real estate into account, by clustering attribute values. Again, this is not targeted to a specific task like explaining the change in aggregate metrics.

The database community has devoted a lot of attention to the problem of processing taxonomical data. For example $[8,22,24,25]$ consider the same or related models. Broadly, they concentrate on the design issues such as query languages, whereas we focus on computational issues and optimization.

There is recent work by Joglekar, Garcia-Molina, and Parameswaran [18], which we call Smart Drill-Down, that like us attempts to "cover" the data. They trade off dual objectives of "covering" the data and "interestingness" with guidance from an analyst. This trade-off is inherently datadependent, because the two objectives are like apples and oranges. The analyst must try some trade-off and rebalance it based on the results. Performing this trade-off effectively probably requires some expertise with data analysis, and the tool is probably not suited to users without such sophistication. In contrast, as we discuss in the introduction, we seek a fully-automated solution for a much less general problem, but this allows us to serve relatively unsophisticated users. Two other differences between their work and ours is that they allow double-counting, which as we discussed is undesirable for our application, and their algorithm is top-down. Indeed, most of OLAP is inherently about top-down exploration. But a top-down algorithm may omit important events in the data. For instance, if you have two adjacent zipcodes in a metro, one with an increase in unemployment, and another with a decrease, the two phenomena could cancel in a way that the net effect on the metro is nearly zero. OLAP on the metro would seem to suggest that nothing interesting is happening within the metro, whereas there may be. There is some work [11, 17, 20, 33] on finding hyper-rectangle based covers for tables. In contrast, our work is about "packing", i.e., we explicitly deal with the double-counting issue.

\subsection{Multistructural Databases}

We are directly inspired by the problem formulations of Fagin et al. [14] and Sarawagi [26]. Fagin et al [14] formulate three operators as optimization problems over lattices-DividE finds balanced partitions of data, Differentiate compares two slices of data along some dimensions to find regions that account for the difference (this is the scenario of Example 1.1 in the introduction), and Discover finds cohesive, well-separated clusters of data. (Sellam and Kersten [32] also work a formulation similar to DIVIDE in the context of designing a query language for exploration.). Discover and DifFERENTIATE are algorithmically similar, differing only in how the weights on the hypernodes are computed. Our algorithms apply to both of these operations. They show that these operators are hard to approximate on hierarchies represented as lattices, and present dynamic programming algorithms that provide approximations for a sequential composition of tree-like or numeric dimensions. Fagin et al. [15] extends these results to a wider class of operators implementable via objective functions that are distributive on the slices, discuss different functions to combine the weights of the output segments, and presents experimental validation for their techniques on a large data set. In a series of papers, Sarawagi et al $[26,28,31]$ discuss information-theoretic formulations that help automate the exploration of datacubes that store aggregates - in the sense of our model, the focus is on designing the objective function (including the weight function). The algorithmic results are similar to the positive results of Fagin et al[14] discussed above.

Our main contribution is to identify practically relevant restrictions of these models, and to supply interesting algorithms. The hardness results in both papers were devastating because the models were overly general (they used lattices instead of a product of a small number of trees). In contrast, their algorithmic results were restrictive as we discuss in Section 6 and Appendix A.

\section{CONCLUSION}

We study the problem of summarizing metric changes across hierarchical dimensions. Our main contribution is a new algorithm called Cascading Analysts that is a significant improvement over prior algorithms for this problem. We apply this algorithm to two widely used business-intelligence tools that help advertisers and website/app developers analyze their core metrics. Studying concrete applications gave us an interesting lens on the computational hardness of the summarization problem. We identified a practically relevant restriction of the previously studied model of hierarchical data to a product space of trees - prior work studied lattices. Without this restriction, the problem on lattices is computationally hard, although, this hardness is relatively uninteresting, i.e., it stems purely from the "dimensionality" of data. Further investigation reveals a more interesting source of hardness - the presence of structures called conflicts that occur in data with three or more dimensions. Fortunately, this source of hardness does not preclude approximation.

Finally, we would like to discuss issues that impact the applicability of the Cascading Analysts algorithm.

- Notice that the runtime of our algorithm is exponential in the number of (hierarchical) dimensions. It is unlikely that our algorithm is efficient when the number of dimensions is larger than 10 (see Remark 1).

- We have focussed our algorithm on the task of explaining metric changes. It is possible that the algorithm is more generally useful by varying the weight function (see Section 2.2). However, there are many cases where the notion of interestingness is either subjective or not clear without interactively inspecting the data. Our algorithm is not applicable to any of these inherently exploratory usecases. (See Section 8.1 for a list of alternatives that apply to these use cases.)

- Recall that the Cascading Analysts algorithm produces the optimal conflict-free solution (Lemma 3.4). It is not optimal if the data has large-weight conflicts, though we 
do provide a worst-case approximation guarantee in the presence of conflicts (see Theorem 3, Remarks 4 and 5), and some empirical evidence that large-weight conflicts rarely arise in practice (see Remark 11), but this evidence is not comprehensive enough to be conclusive. That said, we also show that there is no polynomial time algorithm that achieves optimal results in the presences of conflicts (unless $P=N P$ ).

- Our algorithm produces an explanation of a metric change only in terms of the dimensions and data that are input to it. It is possible that the "true cause" (e.g. a recession) is not directly represented in the data. But an analyst may have the domain knowledge to reason about the true cause. That said, our algorithm can still be a reasonable starting point in the identification of a true cause, and could help to efficiently rule out some causes.

In summary, it is likely that our algorithm is most useful within reporting/decision-making platforms that cater to users who are not necessarily sophisticated in data analysis. It is also likely to be useful as a starting point for some analyses data scientists performs within an organization.

\section{ACKNOWLEDGEMENTS}

We would like to acknowledge Chinmay Karande, Yi Sun, Matthew Paff, Peter Lofgren, and Dave Bogaty who participated in the design of the algorithm. We would like to thank Monica Chawathe, Sunil Kosalge, and Christopher Suter from the AdWords team, and Pew Putthividhya from the Google Analytics Team for their collaborations. Finally, we would like to thank Amir Najmi and D. Sivakumar for their insights.

\section{REFERENCES}

[1] Benchmarking. https://en.wikipedia.org/wiki/Benchmarking.

[2] Explore important insights from your data automatically. https://analytics.googleblog.com/2016/09/explore-importantinsights-from-your.html.

[3] Google adwords. https://en.wikipedia.org/wiki/AdWords.

[4] Google analytics. https://en.wikipedia.org/wiki/Google_Analytics.

[5] Understanding the top movers report in adwords. https://searchengineland.com/google-adwords-new-top-moversreport-aims-to-automate-whats-changed-reporting- 164521.

[6] U.s. census bureau (2005-2015). sex by occupation for the civilian employed population 16 years and over, 2005-2015 american community survey 1-year estimates.

[7] P. K. Agarwal, M. van Kreveld, and S. Suri. Label placement by maximum independent set in rectangles. Computational Geometry, 11(3):209 - 218, 1998

[8] O. Ben-Yitzhak, N. Golbandi, N. Har'El, R. Lempel, A. Neumann S. Ofek-Koifman, D. Sheinwald, E. Shekita, B. Sznajder, and $\mathrm{S}$. Yogev. Beyond basic faceted search. In Proceedings of the international conference on Web search and web data mining, WSDM '08, pages 33-44, New York, NY, USA, 2008. ACM.

[9] P. Berman, B. Dasgupta, S. Muthukrishnan, and S. Ramaswami. Improved approximation algorithms for rectangle tiling and packing (extended abstract). In Proc. 12th ACM-SIAM Symp. on Disc. Alg, pages 427-436, 2001.

[10] G. E. Box and D. Cox. An analysis of transformations, 1964.

[11] S. Bu, L. V. S. Lakshmanan, and R. T. Ng. Mdl summarization with holes. In $V L D B$, pages 433-444, 2005.

[12] K. S. Candan, H. Cao, Y. Qi, and M. L. Sapino. Alphasum: sizeconstrained table summarization using value lattices. In $E D B T$ pages 96-107, 2009.
[13] E. F. Codd, S. B. Codd, and C. T. Salley. Providing OLAP (OnLine Analytical Processing) to User-Analysis: An IT Mandate, 1993.

[14] R. Fagin, R. Guha, R. Kumar, J. Novak, D. Sivakumar, and A. Tomkins. Multi-structural databases. In Proceedings of the twenty-fourth ACM SIGMOD-SIGACT-SIGART symposium on Principles of database systems, PODS '05, pages 184-195, New York, NY, USA, 2005. ACM.

[15] R. Fagin, P. Kolaitis, R. Kumar, J. Novak, D. Sivakumar, and A. Tomkins. Efficient implementation of large-scale multistructural databases. In Proceedings of the 31st International Conference on Very Large Data Bases, VLDB '05, pages 958-969. VLDB Endowment, 2005.

[16] K. E. Gebaly, P. Agrawal, L. Golab, F. Korn, and D. Srivastava. Interpretable and informative explanations of outcomes. PVLDB, pages $61-72,2014$.

[17] F. Geerts, B. Goethals, and T. Mielikinen. Tiling databases. In Discovery Science, pages 278-289, 2004.

[18] M. Joglekar, H. Garcia-Molina, and A. Parameswaran. Smart drilldown: A new data exploration operator. Proc. VLDB Endow., 8(12):1928-1931, Aug. 2015.

[19] S. Khanna, S. Muthukrishnan, and M. Paterson. On approximating rectangle tiling and packing. In Proceedings of the Ninth Annual ACM-SIAM Symposium on Discrete Algorithms, SODA '98, pages 384-393, Philadelphia, PA, USA, 1998. Society for Industrial and Applied Mathematics.

[20] L. V. S. Lakshmanan, R. T. Ng, C. X. Wang, X. Zhou, and T. J. Johnson. The generalized mdl approach for summarization. In $V L D B$, pages $766-777,2002$.

[21] M. Mampaey, N. Tatti, and J. Vreeken. Tell me what i need to know: Succinctly summarizing data with itemsets. In $K D D$, pages 573-581, 2011.

[22] D. Martinenghi and R. Torlone. Querying databases with taxonomies. In J. Parsons, M. Saeki, P. Shoval, C. Woo, and Y. Wand, editors, Conceptual Modeling ER 2010, volume 6412 of Lecture Notes in Computer Science, pages 377-390. Springer Berlin / Heidelberg, 2010. 10.1007/978-3-642-16373-9:27.

[23] D. Pugh. Organization Theory Edited by D.S. Pugh: Selected Readings. Penguin modern management readings. Penguin, 1971.

[24] Y. Qi, K. S. Candan, J. Tatemura, S. Chen, and F. Liao. Supporting olap operations over imperfectly integrated taxonomies. In Proceedings of the 2008 ACM SIGMOD international conference on Management of data, SIGMOD '08, pages 875-888, New York, NY, USA, 2008. ACM.

[25] R. Ramakrishnan and B.-C. Chen. Exploratory mining in cube space. Data Mining and Knowledge Discovery, 15:29-54, 2007. 10.1007/s10618-007-0063-0.

[26] S. Sarawagi. Explaining differences in multidimensional aggregates. In Proceedings of the 25th International Conference on Very Large Data Bases, VLDB '99, pages 42-53, San Francisco, CA, USA, 1999. Morgan Kaufmann Publishers Inc.

[27] S. Sarawagi. User-adaptive exploration of multidimensional data. In $V L D B$, pages $307-316,2000$.

[28] S. Sarawagi. User-Adaptive Exploration of Multidimensional Data. In $V L D B$, pages 307-316. Morgan Kaufmann, 2000.

[29] S. Sarawagi. User-cognizant multidimensional analysis. The VLDB Journal, pages 224-239, 2001.

[30] S. Sarawagi, R. Agrawal, and N. Megiddo. Discovery-driven exploration of olap data cubes. In EDBT, pages 168-182, 1998.

[31] S. Sarawagi and G. Sathe. I3: Intelligent, interactive investigation of olap data cubes. In Proceedings of the 2000 ACM SIGMOD International Conference on Management of Data, SIGMOD '00, pages 589-, New York, NY, USA, 2000. ACM.

[32] T. Sellam and M. L. Kersten. Meet charles, big data query advisor. In $C I D R$ '13, pages $-1-1,2013$.

[33] Y. Xiang, R. Jin, D. Fuhry, and F. F. Dragan. Succinct summarization of transactional databases: an overlapped hyperrectangle scheme. In $K D D$, pages $758-766,2008$.

\section{A BAD EXAMPLES FOR TREE COMPOSITION}

The example below shows that the tree composition algorithm precludes certain combination of data-cubes from being in a candidate solution: 
Example A.1. For our employment example, if we compose the dimensions in a sequence where geography precedes sex, then the following pair of non-overlapping nodes will never be considered in the same solution: (North-east, Male) and (New York, Female). We can only reach both of these nodes by first splitting the space on gender, and then splitting each of subspaces independently on location.

The following example shows that, in the worst-case, the tree composition, even with the best permutation (composition of dimensions), is only a $\Omega\left(n^{1 / 4}\right)$-approximate solution. The example uses two dimensions, and by Theorem 2 , our algorithm is optimal for two dimensions. So, we have a significant worst-case improvement over tree composition even for two dimensions.

Example A.2. Consider an instance of SUMmARIzE with two dimensions, each with the same topology: a star with $\sqrt{m}$ strands (paths), each with $\sqrt{m}$ nodes, and a root. Suppose that the strands are labeled $1 \ldots \sqrt{m}$ from left to right, and the tree-nodes within a strand are labeled $1 \ldots \sqrt{m}$, then we can label each tree-node by a pair (strand index, withinstrand index). Notice that $n=(m+1)^{2}$.

Suppose further that the weights are in $\{0,1\}$. The only lattice nodes with a weight of 1 are the $m$ nodes indexed by the quadruple $(i, j),(j, i)$ for $i \in\{1 \ldots \sqrt{m}\}$ and $j \in$ $\{1 \ldots \sqrt{m}\}$. The optimal solution has value $m$ because none of the non-zero weight nodes overlap - if two nodes overlap in one dimension, then they belong to different strands in the other dimension.

Now, by Observation A.1, for a sequential composition $T_{1}$ followed by $T_{2}$, we cannot pick a pair of lattice nodes that belong to the same strand in dimension $T_{1}$, yielding a solution of size at most $\sqrt{m}$. The argument for the other sequential composition is symmetric, and we have at best a $\sqrt{m}$ approximation. 